\title{
Identification and purification of a soluble region of BubR1: A critical component of the mitotic checkpoint complex
}

\author{
Jongchul Yoon a,1, Yup Kang ${ }^{\text {b,1 }}$, Kyunggon Kim ${ }^{\text {a }}$, Jungeun Park ${ }^{\text {a }}$, \\ Youngsoo Kim ${ }^{\text {a,c,** }}$ \\ ${ }^{a}$ Division of Molecular Genomic Medicine, College of Medicine, Seoul National University, Yongon-Dong, Seoul 110-799, Korea \\ ${ }^{\mathrm{b}}$ Institute for Medical Sciences, Ajou University School of Medicine, Wonchon-dong san 5, Youngtong-gu, Suwon, Kyunggi-do 442-749, Korea \\ ${ }^{\mathrm{c}}$ Cancer Research Institute, College of Medicine, Seoul National University, Yongon-Dong, Seoul 110-799, Korea
}

Received 21 February 2005, and in revised form 14 April 2005

Available online 23 May 2005

\begin{abstract}
The mitotic checkpoint complex (MCC) ensures the fidelity of chromosomal segregation, by delaying the onset of anaphase until all sister chromatids have been properly attached to the mitotic spindle. In essence, this MCC-induced delay is achieved via the inhibition of the anaphase-promoting complex (APC). Among the components of the MCC, BubR1 plays two major roles in the functions of the mitotic checkpoint. First, BubR1 is able to inhibit APC activity, either by itself or as a component of the MCC, by sequestering a APC coactivator, known as Cdc20. Second, BubR1 activates mitotic checkpoint signaling cascades by binding to the centromere-associated protein E, a microtubule motor protein. Obtaining highly soluble BubR 1 is a prerequisite for the study of its structure. BubR1 is a multi-domain protein, which includes a KEN box motif, a mad3-like region, a Bub3 binding domain, and a kinase domain. We obtained a soluble BubR1 construct using a three-step expression strategy. First, we obtained two constructs from BLAST sequence homology searches, both of which were expressed abundantly in the inclusion bodies. We then adjusted the lengths of the two constructs by secondary structure prediction, thereby generating partially soluble constructs. Third, we optimized the solubility of the two constructs by either chopping or adding a few residues at the C-terminus. Finally, we obtained a highly soluble BubR1 construct via the Escherichia coli expression system, which allowed for a yield of $10.8 \mathrm{mg} / \mathrm{L}$ culture. This report may provide insight into the design of highly soluble constructs of insoluble multi-domain proteins.
\end{abstract}

(C) 2005 Elsevier Inc. All rights reserved.

Keywords: BubR1; Structure; Anaphase-promoting complex

In the eukaryotic cell cycle, mitosis consists of six sequential phases: prophase, prometaphase, metaphase, anaphase, telophase, and cytokinesis. During metaphase, two sister chromatids align in the equatorial plane of the dividing cell, while mitotic spindles attach themselves to the kinetochores in the centromeres of the sister chromatids. The mitotic spindle-attached sister chromatids are then separated, and pulled into each spindle pole,

\footnotetext{
* Corresponding author. Fax: +82 27417947.

E-mail address: biolab@snu.ac.kr (Y. Kim).

${ }^{1}$ Two authors contributed equally to this work.
}

thereby completing chromosomal duplication. The mitotic checkpoint (also called the spindle assembly checkpoint) ensures the fidelity of this chromosomal segregation, by delaying the onset of anaphase until all sister chromatids have been properly attached to the mitotic spindle [1].

Mad1 [2], Mad2 [3,4], Bub1 [4], Bub3 [5], and BubR1 $[6,7]$ are some characteristic mitotic checkpoint proteins. These proteins associate with the unattached kinetochores and are involved in the delay in the metaphase/ anaphase transition [4,5,7-9]. In essence, this delay occurs due to inhibition of the anaphase-promoting 
complex (APC), ${ }^{2}$ a multi-subunit E3 ubiquitin ligase, which targets key proteins for destruction, a step necessary for the separation of the sister chromatids [10-13].

The mitotic checkpoint complex (MCC), which includes the BubR1, Bub3, Cdc20, and Mad2 proteins, in nearly equal stoichiometry, has been shown to effect the inhibition of APC activity [14]. The effects of individual checkpoint proteins have also been reported. For example, BubR1 alone has been shown to inhibit APC activity in a much more profound manner than does Mad2 [15]. Two distinct models for the mechanism underlying MCC-induced APC inhibition have been proposed. In the first model, the unattached kinetochore functions as a template which catalyzes the formation of the BubR1Bub3-Mad2-Cdc20 (MCC) complex, which then diffuses away from the kinetochore, to inhibit APC. In the second model, the modification (primarily phosphorylation) of APC by the unattached kinetochore promotes its association with the MCC, thereby resulting in APC inhibition [16].

Among these MCC proteins, BubR1 has been identified as a Mad3/Bub1-related protein kinase, predicated on its homology with the N-terminal domain of budding yeast Mad3, which interacts with Cdc20 [5]. The C-terminal kinase domain of BubR1 is reliant on centromereassociated protein E (CENP-E)-dependent activation [17]. BubR1 also phosphorylates Cdc20 in vitro, and the phosphorylated $\mathrm{Cdc} 20$ consequently precludes its binding to APC, which thereby inhibits mitotic progression [18].

Therefore, BubR1 plays a principal role in monitoring the link between microtubule attachment/tension at the kinetochore, and the movement of the chromosomes [2,19-21]. BubR1 in the mitotic checkpoint plays two principal roles. First, BubR1 can inhibit the activity of APC, either by itself or as a component of the MCC, by sequestering Cdc20 from the APC $[15,16]$. Second, BubR1 activates mitotic checkpoint signaling cascades, by binding to the microtubule motor protein, CENP-E [2,19-21].

The three-dimensional structures of the checkpoint proteins, Mad1, Mad2 [22,23], and Saccharomyces cerevisiae Bub3p [24], have been reported in previous works. The structures of BubR 1 and its homolog-proteins, however, have not yet been reported. The first step in the structural study of BubR1 was to obtain a large amount of soluble protein. However, this proved to be the first bottleneck in the structural study. We attempted to express BubR1 with the Escherichia coli expression system, but it was not easily expressed in soluble form, as BubR1 is a multi-domain protein, which includes structures such as a KEN box motif, a mad3-like region, a Bub3 binding domain, and a kinase domain. Therefore,

\footnotetext{
${ }^{2}$ Abbreviations used: APC, anaphase-promoting complex; MCC, mitotic checkpoint complex; CENP-E, centromere-associated protein E; $\beta \mathrm{ME}, \beta$-mercaptoethanol; $\mathrm{CD}$, circular dichroism; CDD search, conserved domain database searches.
}

we attempted to design soluble BubR1 constructs, using homology searches, secondary structure prediction, and C-terminal residue adjustments. Finally, we did succeed in the generation of soluble BubR 1 constructs, using the E. coli expression system. This report may, then, provide insight into the design of highly soluble constructs of insoluble multi-domain proteins.

\section{Materials and methods}

\section{Cloning of BubRI constructs}

BubR1 constructs, which had been designed via homology searches and secondary structure prediction, were amplified from the full-length BubR 1 gene by PCR. All constructs contained the N-terminal BamHI restriction site, as well as the C-terminal SalI restriction site. The amplified BubR1 constructs were then digested by BamHI and SalI restriction enzymes, and ligated into the pET28a(+) vector (Novagen, USA), which resulted in the cloned BubR1 constructs with both $\mathrm{N}$ - and C-terminal His-tags. The ligated plasmids were then electroporated into E. coli $\mathrm{DH} 5 \alpha$. The cloned BubR1 constructs were verified by double-restriction digestion, and selected clones were finally confirmed via DNA sequencing.

\section{Expression and purification of BubRI constructs}

Cloned BubR1 constructs were subsequently transformed into E. coli BL21(DE3) Codonplus-RIL (Stratagen, USA) for protein over-expression. This substrate was selected to solve the codon bias problem [25]. Overnight culture seeds of BubR1 constructs $(5 \mathrm{ml})$ were then inoculated into $200 \mathrm{ml}$ of LB medium containing kanamycin $(50 \mu \mathrm{g} / \mathrm{ml})$, and allowed to grow at $37^{\circ} \mathrm{C}$. When the optical density of cells at $600 \mathrm{~nm}$ reached between 0.5 and 0.6 , the production of BubR1 proteins was induced via the addition of $0.5 \mathrm{mM}$ IPTG at $21^{\circ} \mathrm{C}$. After overnight induction, the cells were harvested by $10 \mathrm{~min}$ of centrifugation at $4000 \mathrm{~g}$. The cell pellets were then resuspended in $20 \mathrm{mM}$ Tris- $\mathrm{HCl}$ (pH 7.8) containing $200 \mathrm{mM}$ $\mathrm{NaCl}, 1 \mathrm{mM} \beta$-mercaptoethanol ( $\beta \mathrm{ME})$, and protease inhibitor cocktail (Roche, Switzerland). The resuspended cells were homogenized by ultra-sonication and supernatants were separated by $25 \mathrm{~min}$ of centrifugation at $15,000 \mathrm{~g}$. The expression profiles of the BubR1 constructs were visualized by SDS-PAGE.

To purify the BubR1, the cell lysate supernatants were loaded onto Ni-NTA resin (Qiagen, USA). Elution was then performed with $50-500 \mathrm{mM}$ concentration gradient of imidazole. The purified BubR1 was identified by SDS-PAGE and then dialyzed against $20 \mathrm{mM}$ sodium phosphate ( $\mathrm{pH} 7.5)$, containing $1 \mathrm{mM} \beta \mathrm{ME}$.

All BubR1 constructs $(1-310,1-312,1-314,1-316$, $1-318,1-320,1-322,1-324,1-326,1-328$, and 1-330, in 
which the two residue numbers linked by a hyphen represent cloned constructs throughout the text) were purified to determine the soluble fraction yield with $200 \mathrm{ml}$ cultures, but selected BubR1 constructs (1-286 and 1-320) were then employed for large-scale protein purification with $4.2 \mathrm{~L}$ cultures.

\section{Homology search and secondary structure prediction}

The selection of constructs for the cloning of BubR1 was based on comparative amino acid sequence analyses, secondary structure prediction, and functional studies of BubR1. Comparative sequence analysis was performed using BLAST [26], and secondary structure prediction involved the GOR4 consensus program, including the GOR4 [27], HNN [28], PHD [29], Predators [30], and SIMPA96 [31] prediction programs. The GOR4 consensus program reduced prediction errors and provided reliable predictions with regard to secondary structure.

\section{Circular dichroism spectrometry}

Far-UV (190-260 nm) circular dichroism (CD) spectra were measured with a Jasco J-715 spectrometer [32]. The CD experiment was performed at $0.5 \mathrm{~nm}$ intervals, in a $0.1 \mathrm{~cm}$ quartz cuvette, at $20^{\circ} \mathrm{C}$. The spectra of the BubR1 constructs 1-286 and 1-320 are expressed as an average of three scans, to compensate for measurement errors and buffer absorbance. The protein concentration was $0.25 \mathrm{mg} / \mathrm{ml}$, buffered by $5 \mathrm{mM}$ sodium phosphate ( $\mathrm{pH}$ 7.4). Spectra were digitized by recording in millidegree increments, and these values were then converted to per residue molar absorption, expressed in $m d e g / \mathrm{M} / \mathrm{cm}$. Experimental CD spectra were fitted to estimate the composition of the secondary structures, using the COTINLL program, at the DICHROWEB website [31,33].

\section{MALDI-TOF and MALDI-TOF/TOF analysis}

Smaller bands than expected for the target construct (residues 1-320) appeared during BubR1 purification. Thus, we cut out the smaller minor bands with SDSPAGE, to ascertain whether or not they represented proteolytically digested forms from the correctly-sized target protein. Interesting SDS-PAGE spots were excised from the stained gels, and the gel spots were sequentially destained, reduced, alkylated, and hydrolyzed, using modified porcine trypsin (Promega, Southhampton, UK), as previously described $[34,35]$. The extracted in-gel digested peptides were then identified via peptide mass fingerprinting using MALDI-TOF MS, and the identified peptides were subsequently sequenced via MALDI-TOF/TOF tandem mass spectrometry (MS/MS) (Applied Biosystems, USA) at the Seoul National University College of Medicine, in Seoul, Korea [36]. Database searches were carried out using the search program, MASCOT, developed by Matrix Science Ltd (access is available on http:// www.matrix.science.com), and the ExPASy Molecular Biology Server at SWISS-PROT (http://www.expasy.ch).

\section{Results and discussion}

\section{Preliminary expression of BubR1 domains}

Human BubR1 is a multi-domain protein consisting of 1050 residues [5]. The domains of human BubR1 were easily identified with alignment to Xenopus BubR1, which is a homolog of human BubR1 [17], using BLAST software [26]. It turned out that human BubR 1 consists of a KEN box motif (residues 26-28), a mad3-like region (residues 52-204), a Bub3 binding domain (residues 392433), and a kinase domain (749-1018).

Human BubR1 as a multi-domain protein cannot be easily expressed with the standard $E$. coli expression system. Thus, we examined several distinctive BubR1 domain by sequence homology and conserved domain database searches (CDD search) using BLAST software [26]. Protein expression was pre-examined for the selected BubR1 domains garnered in the BLAST search, namely, the residues 1-207 (KEN box motif and mad3like region), 50-207 (mad3-like region only), 1-460 (KEN box motif, mad3-like region, and Bub3-binding domain), 50-460 (mad3-like region and Bub3-binding domain), and 755-1023 (C-terminal kinase domain). The expression patterns of these domains are shown in Fig. 1. The constructs without N-terminal regions (residues 50-207 and 50-460) were expressed in inclusion bodies, similarly to those with N-termini (residues 1-207 and 1-460). Kinase domains (residues 755-1023) could not be expressed, even in the inclusion bodies.

\section{Rational design of soluble BubRI constructs}

After expressing the selected domains obtained in the BLAST search, we assessed the solubility of the BubR1 constructs designed by secondary structure prediction. The BubR1 constructs, 1-221, 1-238, 1-260, 1-275, 1-286, 1-320, 1-486, 50-221, 50-238, 50-260, 50-275, 50-286, and $50-320$, were designed via secondary structure prediction, based on the preliminary expression data obtained in the previous section, in which the constructs, 1-207, 50-207, 1-460, and 40-460, resulted in inclusion body expression (Fig. 2). The final amino acids in the constructs were chosen such that the residues $(221,241,260$, 275,286 , and 320 ) were positioned in the random coil regions of the predicted secondary structures (Fig. 2). These constructs commonly contain the region (208-381) which exists between the Mad3-like region (50-207), and the Bub3-binding region (382-456), with the exception of construct 1-486. 

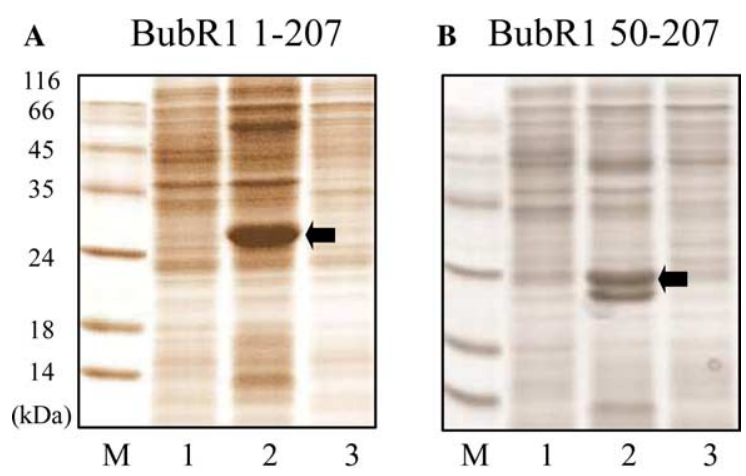

C BubR1-460

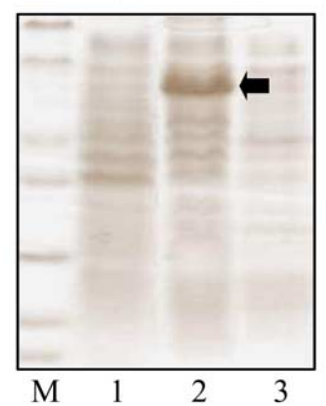

D BubR1 50-460

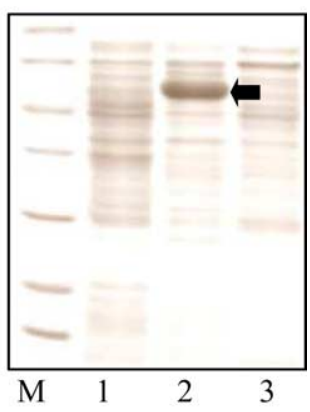

E BubR1 KD

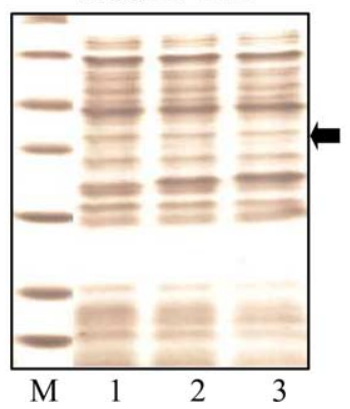

Fig. 1. Preliminary expression of BubR 1 domains. Expression patterns represent (A) BubR1 construct 1-207, (B) BubR1 construct 50-207, (C) BubR1 construct 1-460, (D) BubR1 construct 50-460, and (E) BubR1 construct 755-1023 (kinase domain). The arrows represent target proteins throughout the figure legends. M, 1,2, and 3, located at the bottom of the gel, represent molecular weight markers, uninduced cell lysates, induced cell lysates, and induced supernatants, respectively. $\mathrm{KD}$ represents the kinase domain. The numbers on the vertical column of (A) represent the molecular weight of the molecular weight marker. This marker was also used for all the other SDS-PAGE gels.

Secondary structure prediction was the main consideration for all construct designs in this section. All constructs were ligated in the random coil regions suggested in secondary structure prediction (Fig. 2), with one exception. The construct 1-286 was cut one residue ahead of the beginning of the $\alpha$-helix, as Leu 287 utilizes one of the deficient amino-acyl t-RNA synthetases in the $E$. coli system. Among the five programs utilized, only the HNN program predicted that the residue 486 would be located in the $\alpha$-helix, whereas the other four programs predicted that the residue 486 would be located in the random coil region. Q487 is located in the random coil but is a bulky residue. Thus, residue 486 was selected for the C-terminus of construct 1-486.

Those 13 constructs were evaluated with regard to their solubility. Some of the constructs (1-221, 1-238, 1-260, 1-275, 1-286, 1-320, and 1-486) were shown, but soluble expressions were detected only in the 1-286 and 1-320 constructs (Fig. 3). The constructs without the 50 $\mathrm{N}$-terminal residues were all expressed in inclusion bodies (data not shown). It would appear that the N-terminal region does, indeed, play a primary role in soluble expression.

By this rational design, using secondary structure prediction programs, we generated two soluble constructs from the 13 constructs in which the C-termini were positioned in the random coil region. It turned out that the previous expression in the inclusion bodies could be improved to partial solubility by adjusting the C-terminal regions, based on the secondary structure predictions.

\section{Purification of the constructs 1-286 and 1-320}

The constructs 1-286 and 1-320, located on the vector pET28a(+), were expressed in E. coli BL21(DE3) Codonplus-RIL, and were purified via Ni-NTA affinity chromatography (Figs. 4A and B). The purified 1-286 was found to be unstable at $3 \mathrm{mg} / \mathrm{ml}$ during concentration, whereas the 1-320 construct was determined to be stable at a concentration of $10 \mathrm{mg} / \mathrm{ml}$. The purified $1-320$ contained smaller minor contaminated bands, which were below the target size (Fig. 4B). Therefore, we used anion ion exchange chromatography with Q-Sepharose, to eliminate the undesired smaller proteins, as the 1-320 construct had a $\mathrm{p} I$ value of 5.4. However, the minor bands were still found to be co-eluted with the 1-320 construct (Fig. 4C), at an $\mathrm{NaCl}$ gradient of $150-300 \mathrm{mM}$. We assumed, then, that the smaller contaminated bands might be degraded forms of the 1-320 construct. We detected two smaller minor bands to have been co-eluted with the target construct 1-320 (Fig. 4C), and these bands were subjected to MALDI-TOF MS for peptide mass fingerprinting and, subsequently, to MALDI-TOF/ TOF MS/MS for peptide sequencing (data not shown). The two smaller minor bands were determined to be proteolytically digested fragments from the target 1-320 construct, the C-termini of which were the residues Arg224 and Arg-271. The Arg-224 and Arg-271 residues are located near the end of the random coil region (Fig. 2).

Presumably, it will be necessary to perform gel filtration chromatography to get rid of the smaller minor contaminant bands of the construct 1-320 in the next step, to purify only the correctly-sized band for the construct 1-320. Beforehand, it will be crucial to minimize proteolytic degradation by adding protease inhibitor cocktail from the cell lysis step during purification. 
MAAUKKEGGAL SEAMSLEGDEWELSKE MUQPLRQGR I MST LQGALAQESA

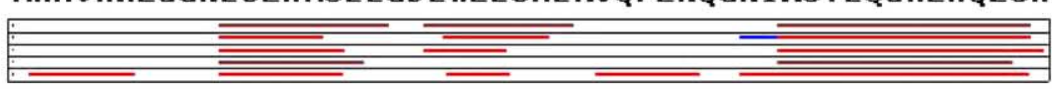

CNHT LQQQKRAFEYE I RFYT GNDPLDUWDRY I SWTEQNYPQGEKESNMST

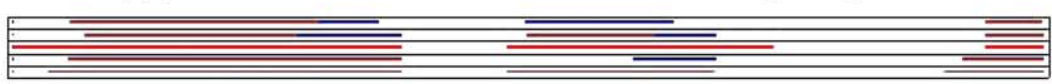

LLERAUEALQGE KRYYSDPRFLNLWLKLGRLCNEPLDHYSYLHNQGIGUS

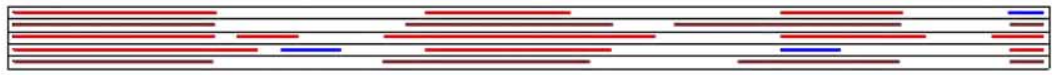

LAQF Y I S WAE EYE ARE NFRKADA I F QEG IQQQKAEPLERLQSQHRQF QQARU

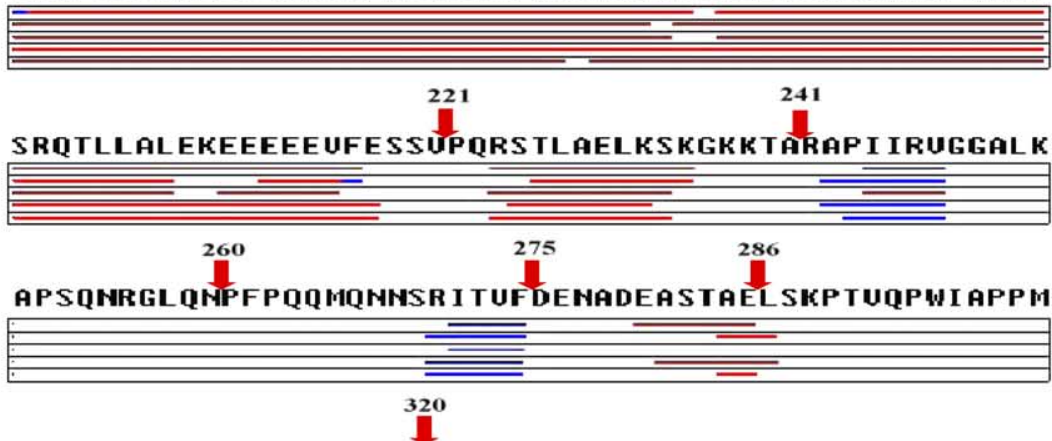

PRAKENELQAGPUNTGRSLEHRPRGTASLIAUPAULPSFTPYUEETAQQ

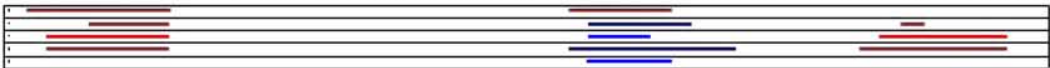

PUMT PCK IEPS I MH I LSTRKPGKEEGDSLQRUQSHQQASEEKKEKHMYCK

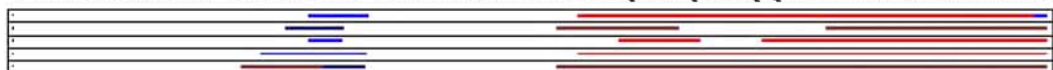

EK I Y AGUGEF SFEE I RAEUF RKKLKEQREAELL TSAEKRAEMQKQ I EEME

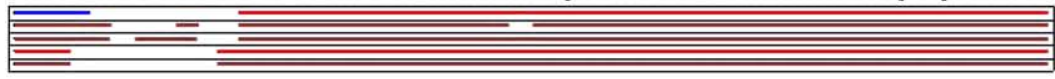

486

KKLKE IQTTQQERT TDQQEE TMPTKETTKLQIASESQK I PGMTLSS SUCQ

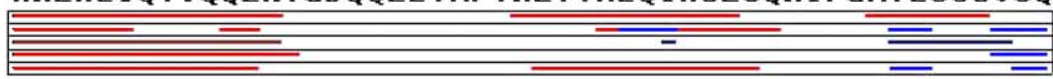

$\square: \alpha$-helix $\square: \beta$-sheet $\square$ : Random coil region

Fig. 2. Prediction of secondary structure for the residues 1-500. Secondary structures of residues 1-500 were predicted by the GOR4 consensus program, including GOR4 [27], HNN [28], PHD [29], Predators [30], and SIMPA96 [31], which are shown in sequence from top to bottom. The arrows indicate the $\mathrm{N}$-termini of the BubR1 constructs, the residues of which belong to the notified secondary structures seen in the figure.

Optimizing soluble expression of the construct 1-320

To further maximize the solubility of the partially soluble 1-320 construct, we refined the domain stability by either chopping or adding a few residues at the C-terminus of the 1-320 construct. The resultant constructs, 1-310, $1-312,1-314,1-316,1-318,1-322,1-324,1-326,1-328$, and $1-330$, were designed to further assess the soluble expression of the new constructs in which the template construct, 1-320, was used as a control. Similar yields of soluble expression were obtained from constructs 1-310 to
1-318, whereas increased yields of soluble expression were detected when using the 1-322 to 1-328 constructs (Fig. 5). Quantitation of soluble expression was performed with the 12 constructs, using the same cell mass (Table 1). Interestingly, the soluble yield of the 1-330 construct decreased dramatically (Table 1). It appears that the low yield of the soluble 1-330 construct might result from the disruption of the beginning of the $\beta$-sheet (predicted by GOR4) or the $\alpha$-helix (predicted by HNN, PHD, Predator, and SIMPA96) (Fig. 6), in which the C-terminus of construct 1-330 was terminated in the middle of either the 
A

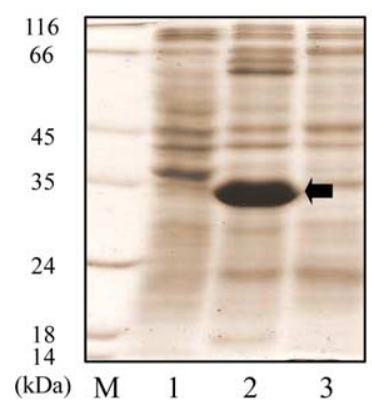

B BubR1 1-238

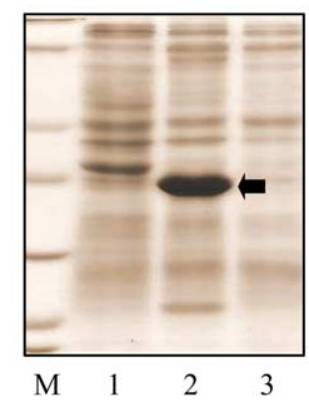

C BubR1 1-260

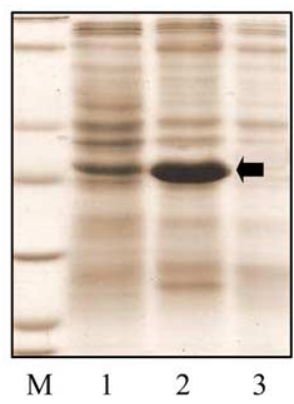

D BubR1 1-275

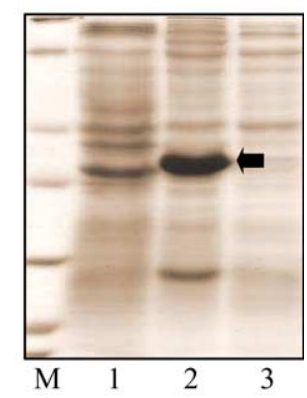

E BubR1 1-286

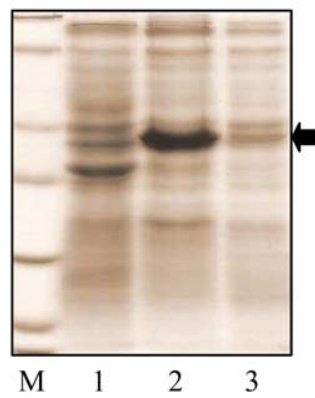

F BubR1 1-320

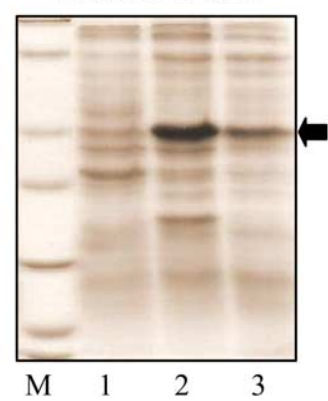

G BubR1 1-486

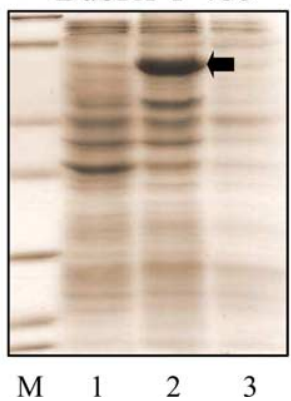

Fig. 3. Expression patterns of soluble BubR1 constructs. Figures represent (A) BubR1 construct 1-221, (B) BubR1 construct 1-238, (C) BubR1 construct 1-260, (D) BubR1 construct 1-275, (E) BubR1 construct 1-286, (F) BubR1 construct 1-320, and (G) BubR1 construct 1-486. All constructs lacking residues 1-50 were expressed in inclusion bodies (data not shown). The gel notations are the same as in Fig. 1.

\section{A BubR1 1-286}

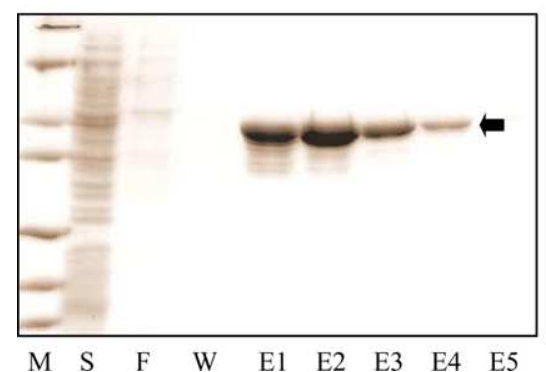

B BubR1 1-320

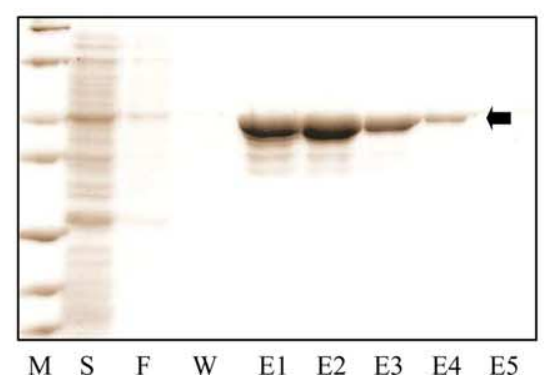

C BubR1 1-320

C M Salt gradient

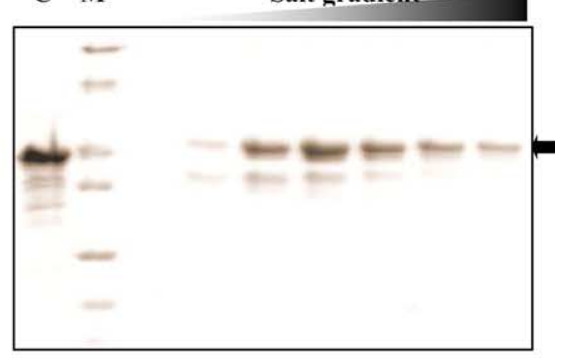

Fig. 4. Purification of the constructs BubR1 1-286 and BubR1 1-320. (A) Ni-NTA affinity chromatography of the construct BubR1 1-286. (B) NiNTA affinity chromatography of the construct BubR1 1-320. (C) Q-Sepharose anion exchange chromatography of the construct 1-320. M, S, F, W, and E1-E5 represent molecular marker, cell supernatant, flow through, wash fraction, and fraction eluted by imidazole gradient. Smaller minor contaminant bands were co-eluted with the BubR1 construct 1-320 in (C). C, M, and salt gradient represent loading fraction, molecular weight marker, and $\mathrm{NaCl}$ gradient eluted fractions.

$\beta$-sheet or the $\alpha$-helix. This result suggested that the disruption of the secondary structure at the end region of the constructs (presumably, also located at the N-terminus) should be avoided to ensure optimized solubility in the domain constructs.

The differential yield of solubility between constructs 1-328 and 1-330 led us to chop out or add a few residues to the 1-207 construct (Fig. 1), to optimize its solubility. In contrast to the 1-320 construct, the C-terminal region of the 1-207 construct spans a long $\alpha$ helix, from residues 149 to 218 (Fig. 2). Thus, we were not able to rationally choose an appropriate residue for the $\mathrm{C}$-termini for new constructs, as any $\mathrm{C}$-terminal residue would be positioned in the middle of the $\alpha$ helix. Therefore, constructs 1-200, 1-204, and 1-212 were chosen arbitrarily, and used for solubility evaluations. The 1-200 and 1-204 constructs were better expressed in soluble forms, while construct 1-212 continued to be expressed in inclusion bodies, identically to construct 1-207. Surprisingly, the chopping out or adding of a few residues at the C-terminus resulted in a minor improvement of the soluble yield of construct 1207, although the C-terminus had been chosen in the middle of the secondary structure, in this case the $\alpha$ - 


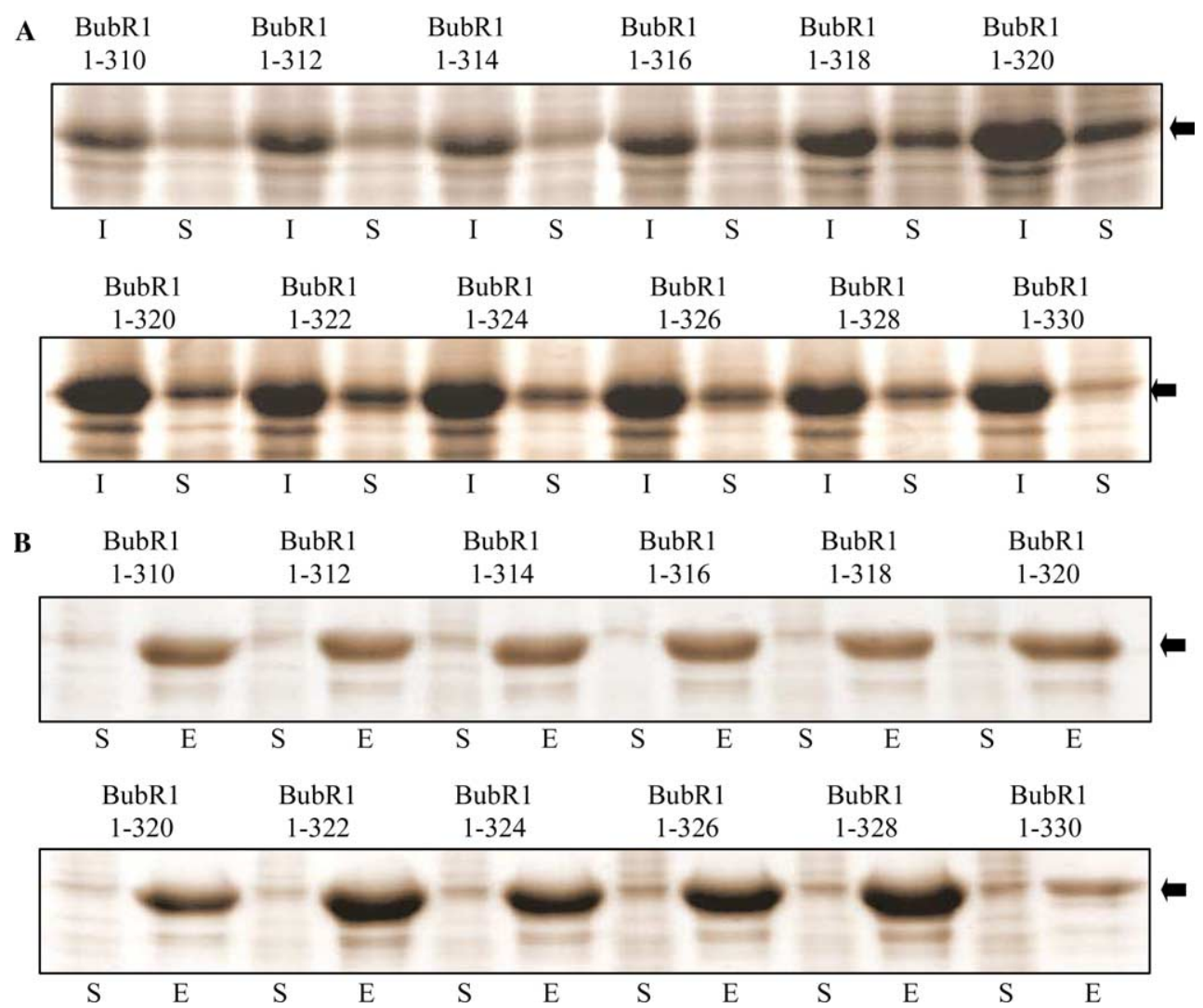

Fig. 5. Optimizing solubility of the construct BubR1 1-320. (A) Expression patterns of the BubR1 constructs 1-310, 1-312, 1-314, 1-316, 1-318, 1-320, 1-322, 1-324, 1-326, 1-328, and 1-330. Total yields of each BubR1 construct increased between constructs BubR1 1-320 and 1-328. I and S represent induced cell lysates and induced cell supernatants, respectively. (B) Purification yields of the BubR1 1-310 to 1-330 constructs (see also Table 1). Similar purification yields for soluble proteins were obtained from the BubR1 constructs 1-310 to 1-318, but increased yields were observed when the BubR1 constructs 1-322 to 1-328 were used, as compared to the BubR1 construct 1-320. S and E represent induced cell supernatant and eluted fraction at $500 \mathrm{mM}$ imidazole concentrations, respectively.

Table 1

Quantification of soluble yields for the BubR1 constructs

\begin{tabular}{lllc}
\hline Constructs & $\begin{array}{l}\text { Soluble } \\
\text { yield }(\mathrm{mg} / \mathrm{L})\end{array}$ & Constructs & $\begin{array}{l}\text { Soluble } \\
\text { yield }(\mathrm{mg} / \mathrm{L})\end{array}$ \\
\hline BubR1 1-310 & 4.4 & BubR1 1-320 & 4.3 \\
BubR1 1-312 & 4.1 & BubR1 1-322 & 10.4 \\
BubR1 1-314 & 4.3 & BubR1 1-324 & 8.4 \\
BubR1 1-316 & 4.3 & BubR1 1-326 & 9.2 \\
BubR1 1-318 & 4.1 & BubR1 1-328 & 10.8 \\
BubR1 1-320 & 4.3 & BubR1 1-330 & 1.5 \\
\hline
\end{tabular}

Soluble yield is based on $1 \mathrm{~L}$ culture. Protein concentrations were measured by Bradford method.

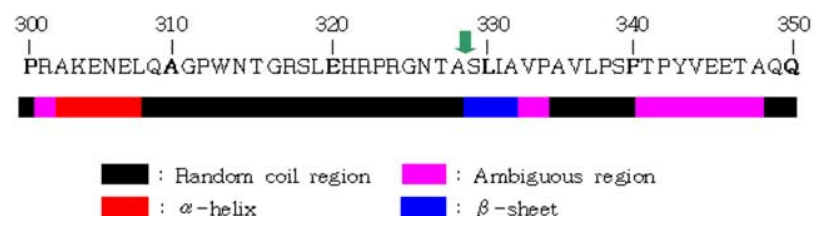

Fig. 6. Secondary structure prediction of the residue 300-350. The arrow indicates the beginning (residue 329) of a $\beta$-sheet. There, the $\mathrm{C}$ terminus of residue 330 disrupted the newly begun $\beta$-sheet, resulting in decreasing solubility of the BubR1 construct 1-330. This prediction was provided by the GOR4 prediction program [27]. helix (Fig. 7). However, in contrast to what was observed withconstruct 1-320, the improvement of the solubility of construct 1-207 was not as dramatic as that of construct 1-320, which might be due to the fact that the C-terminus of construct 1-207 is positioned in the region of the $\alpha$-helix. Even so, the solubility of insoluble constructs should be influenced radically by a few residues at the $\mathrm{N}$ - or $\mathrm{C}$-terminus. Therefore, the solubility of the domain construct should be assessed by the adjustment of a few residues at either the N-or C-terminus, to determine the maximum yield of protein expression.

\section{Circular dichroism for BubRI constructs}

$\mathrm{CD}$ is useful for the determination of proteins' secondary structure, as it allows the researcher to visually verify the differential manner in which a protein absorbs left- and right-hand circular polarized lights [32]. To examine the compositions of the secondary structures of the BubR1 constructs, we obtained the CD spectra of constructs 1-286 and 1-320. The two CD spectra evidenced characteristics typical of $\alpha$-helix-containing pro- 


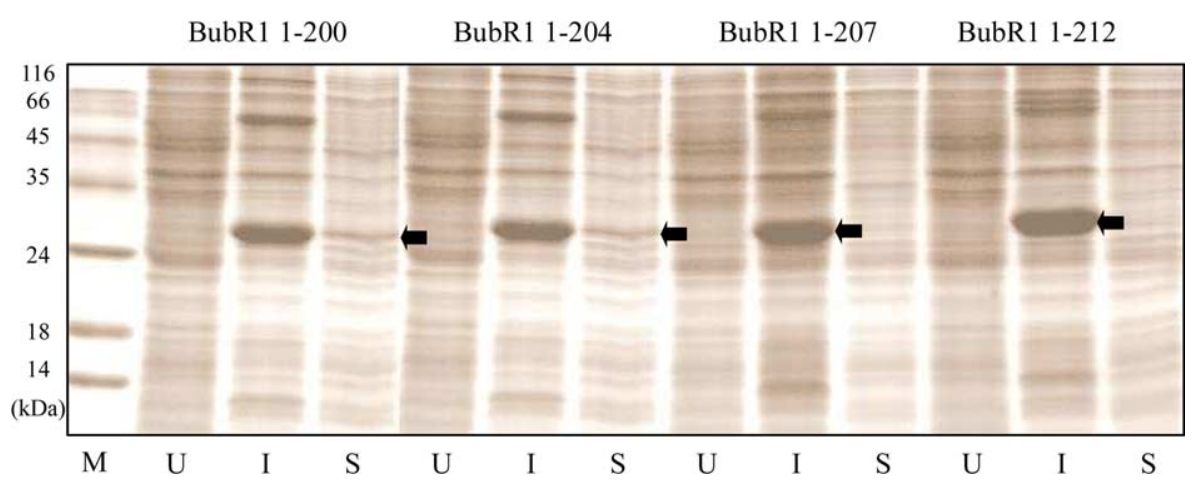

Fig. 7. Optimizing soluble expressions of the construct BubR1 1-207. The BubR1 constructs, 1-200, 1-204, and 1-212, were examined with regard to their soluble expression. The construct, BubR1 1-207, was used for expression control. U, I, and S represent uninduced cell lysates, induced cell lysates, and induced cell supernatants, respectively.

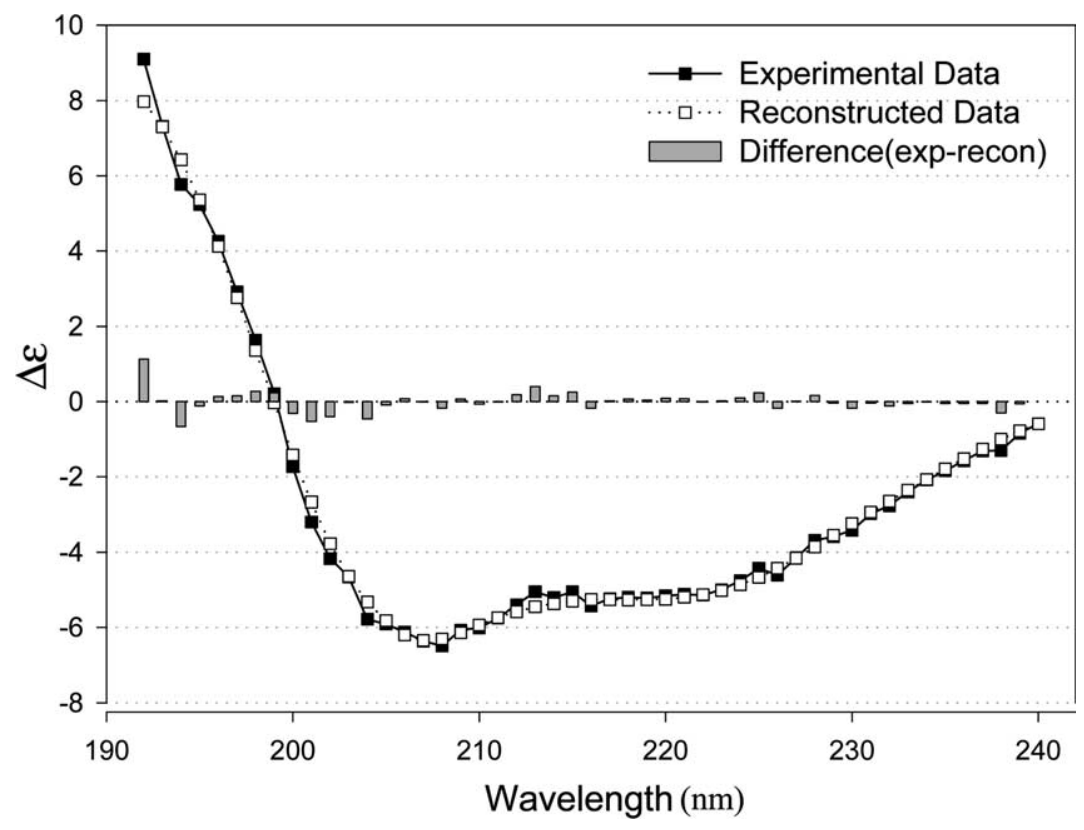

Fig. 8. Circular dichroism spectrum for construct 1-320. The vertical axis (Delta Epsilons $\Delta \varepsilon$ ) represents per residue molar absorption in mdeg/M/cm. The horizontal axis shows wavelength in nanometer.

teins, exhibiting a negative band at $210 \mathrm{~nm}$, and a shoulder at approximately $220 \mathrm{~nm}$ (data is presented only for construct 1-320) (Fig. 8). The experimental CD spectrum of construct 1-320 was fitted using the CONTINLL program $[37,38]$ in the the DICROWEB website [33], to assess the composition of the secondary structure. The CONTINLL program calculated that the secondary structural composition of construct 1-320 was composed of $46.7 \% \alpha$-helix, $1.9 \% \beta$-sheet, $10.8 \%$ turns, and $40.6 \%$ random coil, whereas the GOR program for secondary structure prediction predicted that it consisted of $47.8 \% \alpha$-helix, $7.8 \% \beta$-sheet, and $44.4 \%$ random coil (Fig. 2). Interestingly, the values for the $\alpha$-helix and those for the random coil were predicted fairly accurately by both the CONTINLL and the GOR programs, whereas these programs did not do such a good job in predicting the values associated with the $\beta$-sheet. In retrospect, it turned out to be quite appropriate that our construct design had been predicated on the secondary structure prediction programs (Fig. 2), considering that the secondary structure prediction programs accurately matched the results of the $\mathrm{CD}$ experiments done in this study.

\section{Acknowledgments}

This work was supported by Grant R02-2003-00010010-0 from the Basic Research Program of the Korea Science and Engineering Foundation, and also supported in part by Grant No. 03-2003-005 from the SNUH Research Fund to Y. Kim. 


\section{References}

[1] C.L. Rieder, R.W. Cole, A. Khodjakov, G. Sluder, The checkpoint delaying anaphase in response to chromosome mono-orientation is mediated by unattached kinetochores, J. Cell Biol. 130 (1995) 941-948.

[2] R.H. Chen, A. Shevchenko, M. Mann, A.W. Murray, Spindle checkpoint protein Xmad1 recruits Xmad2 to unattached kinetochores, J. Cell Biol. 143 (1998) 283-295.

[3] Y. Li, R. Benezra, Identification of a human mitotic checkpoint gene : hsMAD2, Science 274 (1996) 246-248.

[4] S.S. Taylor, F. McKeon, Kinetochore localization of murine Bub1 is required for normal mitotic timing and checkpoint response to spindle damage, Cell 89 (1997) 727-735.

[5] S.S. Taylor, E. Ha, F. McKeon, The human homologue of Bub3 is required for kinetochore localization of Bub1 and a Mad3/Bub1related protein kinase, J. Cell Biol. 142 (1998) 1-11.

[6] G.K. Chan, B.T. Schaar, T.J. Yen, Characterization of the kinetochore binding domain of CENP-E reveals interactions with the kinetochore proteins CENP-F and hBUBR1, J. Cell Biol. 143 (1998) 49-63.

[7] G.K. Chan, S.A. Jablonski, V. Sudakin, J.C. Hittle, T.J. Yen, Human BUBR1 is a mitotic checkpoint kinase that monitors CENP-E functions at kinetochores and binds the cyclosome/APC, J. Cell Biol. 146 (1999) 941-954.

[8] R.H. Chen, J.C. Waters, E.D. Salmon, A.W. Murray, Association of spindle assembly checkpoint component XMAD2 with unattached kinetochores, Science 274 (1996) 242-246.

[9] J.V. Shah, D.W. Cleveland, Waiting for anaphase: Mad2 and the spindle assembly checkpoint, Cell 103 (2000) 997-1000.

[10] R.W. King, J.-M. Peters, S. Tugendreich, M. Rolfe, P. Hieter, M.W. Kirschner, A 20S complex containing CDC27 and CDC16 catalyses the mitosis-specific conjugation of ubiquitin to cyclin B, Cell 81 (1995) 279-288.

[11] R.W. King, R.J. Deshaies, J.M. Peters, M.W. Kirschner, How proteolysis drives the cell cycle, Science 274 (1996) 1652-1659.

[12] V. Sudakin, D. Ganoth, A. Dahan, H. Heller, J. Hershko, F.C. Luca, J.V. Ruderman, A. Hershko, The cyclosome, a large complex containing cyclin-selective ubiquitin ligase activity, targets cyclins for destruction at the end of mitosis, Mol. Biol. Cell 6 (1995) 185-197.

[13] A. Hershko, A. Ciechanover, The ubiquitin system, Annu. Rev. Biochem. 67 (1998) 425-479.

[14] V. Sudakin, G.K. Chan, T.J. Yen, Checkpoint inhibition of the APC/C in HeLa cells is mediated by a complex of BUBR1, BUB3, CDC20, and MAD2, J. Cell Biol. 154 (2001) 925-936.

[15] Z. Tang, R. Bharadwaj, B. Li, H. Yu, Mad2-independent inhibition of APC Cdc20 by the mitotic checkpoint protein BubR1, Dev. Cell 1 (2001) 227-237.

[16] R. Bharadwaj, H. Yu, The spindle checkpoint, aneuploidy, and cancer, Oncogene 23 (2004) 2016-2057.

[17] Y. Mao, A. Abrieu, D.W. Cleveland, Activating and silencing the mitotic checkpoint through CENP-E-dependent activation/inactivation of BubR1, Cell 114 (2003) 87-98.

[18] G. Fang, Checkpoint protein BubR1 acts synergistically with Mad2 to inhibit anaphase-promoting complex, Mol. Biol. Cell 13 (2002) 755-766.

[19] B.T. Schaar, G.K. Chan, P. Maddox, E.D. Salmon, T.J. Yen, CENP-E function at kinetochores is essential for chromosome alignment, J. Cell Biol. 139 (1997) 1373-1382.
[20] K.W. Wood, R. Sakowicz, L.S. Goldstein, D.W. Cleveland, CENP$\mathrm{E}$ is a plus end-directed kinetochore motor required for metaphase chromosome alignment, Cell 91 (1997) 357-366.

[21] X. Yao, A. Abrieu, Y. Zheng, K.F. Sullivan, D.W. Cleveland, CENP-E forms a link between attachment of spindle microtubules to kinetochores and the mitotic checkpoint, Nat. Cell Biol. 2 (2000) 484-491.

[22] X. Luo, G. Fang, M. Coldiron, Y. Lin, H. Yu, M.W. Kirschner, G. Wagner, Structure of the Mad2 spindle checkpoint protein and its interaction with Cdc20, Nat. Struct. Biol. 7 (2000) 224-229.

[23] L. Sironi, M. Mapelli, S. Knapp, A.D. Antoni, K.T. Jeang, A. Musacchio, Crystal structure of the tetrameric Mad1-Mad2 core complex: implication of a 'safety belt' binding mechanism for the spindle checkpoint, EMBO J. 21 (2002) 2496-2506.

[24] N.A. Larsen, S.C. Harrison, Crystal structure of the spindle assembly checkpoint protein Bub3, J. Mol. Biol. 344 (2004) 885-892.

[25] C. Gustafsson, S. Govindarajan, J. Minshull, Codon bias and heterogous protein expression, Trends Biotechnol. 22 (2004) 346-353.

[26] S.F. Altschul, T.L. Madden, A.A. Schaffe, J. Zhang, Z. Zhang, W. Miller, D.J. Lipman, Gapped BLAST and PSI-BLAST: a new generation of protein database search programs, Nucleic Acids Res. 1 (1997) 3389-3402.

[27] J. Garnier, J.F. Gibrat, B. Robson, GOR secondary structure prediction method version IV, Methods Enzymol. 266 (1996) 540-553.

[28] Y. Guermeur, Combinaison de classifieurs statistiques, Application a la prediction de structure secondaire des proteines, Ph.D. Thesis.

[29] B. Rost, C. Sander, Prediction of protein secondary structure at better than 70\% accuracy, J. Mol. Biol. 232 (1993) 584-599.

[30] D. Frishman, P. Argos, Incorporation of non-local interactions in protein secondary structure prediction from the amino acid sequence, Protein Eng. 9 (1996) 133-142.

[31] J.M. Levin, B. Robson, J. Garnier, An algorithm for secondary structure determination in proteins based on sequence similarity, FEBS Lett. 205 (1986) 303-308.

[32] W.C. Johnson Jr., Protein secondary structure and circular dichroism: a practical guide, Proteins: structure, function, and genetics 7 (1990) 205-214.

[33] L. Whitmore, B.A. Wallace, DICHROWEB: an online server for protein secondary structure analyses from circular dichroism spectroscopic data, Nucleic Acids Res. 32 (2004) 668-673.

[34] J. Rosenfeld, J. Capdevielle, J.C. Guillemot, P. Ferrara, In-gel digestion of proteins for internal sequence analysis after one- or twodimensional gel electrophoresis, Anal. Biochem. 203 (1992) 173-179.

[35] M. Shevchenko, M. Wilm, O. Vorm, M. Mann, Mass spectrometric sequencing of proteins silver-stained polyacrylamide gels, Anal. Chem. 68 (1996) 850-858.

[36] C. Scheler, S. Lamer, Z. Pan, X.P. Li, J. Salnikow, P. Jungblut, Peptide mass fingerprint sequence coverage from differently stained proteins on two-dimensional electrophoresis patterns by matrix assisted laser desorption/ionization-mass spectrometry (MALDIMS), Electrophoresis 19 (1998) 918-927.

[37] S.W. Provencher, J. Glockner, Estimation of globular protein secondary structure from circular dichroism, Biochemistry 20 (1981) $33-37$.

[38] I.H.M. Van Stokkum, H.J.W. Spoelder, M. Bloemendal, R. Van Grondelle, F.C.A. Groen, Estimation of protein secondary structure and error analysis from CD spectra, Anal. Biochem. 191 (1990) 110-118. 